\title{
Acute Aerobic Exercise Based Cognitive and Motor Priming: Practical Applications and Mechanisms
}

\author{
Terence A. Moriarty ${ }^{1,2 *}$, Christine Mermier ${ }^{1}$, Len Kravitz ${ }^{1}$, Ann Gibson ${ }^{1}$, Nicholas Beltz ${ }^{3}$ \\ and Micah Zuhl1,4
}

\begin{abstract}
'Department of Health, Exercise, and Sports Sciences, The University of New Mexico, Albuquerque, NM, United States, ${ }^{2}$ Department of Kinesiology, University of Northern lowa, Cedar Falls, IA, United States, ${ }^{3}$ Department of Kinesiology, University of Wisconsin-Eau Claire, Eau Claire, WI, United States, ${ }^{4}$ School of Health Sciences, Central Michigan University, Mount Pleasant, MI, United States
\end{abstract}

\section{OPEN ACCESS}

Edited by:

Matteo De Marco,

The University of Sheffield,

United Kingdom

Reviewed by:

William Richard Staines, University of Waterloo, Canada

Chia-Liang Tsai, National Cheng Kung University,

Taiwan

*Correspondence:

Terence A. Moriarty

terence.moriarty@uni.edu

Specialty section:

This article was submitted to

Neuropsychology,

a section of the journal

Frontiers in Psychology

Received: 03 September 2019 Accepted: 27 November 2019 Published: 12 December 2019

Citation:

Moriarty TA, Mermier C, Kravitz L, Gibson A, Beltz N and Zuhl M (2019)

Acute Aerobic Exercise Based Cognitive and Motor Priming:

Practical Applications and Mechanisms.

Front. Psychol. 10:2790. doi: 10.3389/fpsyg.2019.02790
Acute exercise stimulates brain regions involved in motor and cognitive processes. Recent research efforts have explored the benefits of aerobic exercise on brain health and cognitive functioning with positive results reported for both healthy and neurocognitively impaired individuals. Specifically, exercise positioned near therapeutic (both behavioral and physical) activities may enhance outcomes associated with treatment outcomes (e.g., depression or motor skill) through neural plasticity promoting mechanisms (e.g., increased brain flow and oxygenation). This approach has been termed "exercise priming" and is a relatively new topic of exploration in the fields of exercise science and motor control. The authors report on physiological mechanisms that are related to the priming effect. In addition, parameters related to the exercise bout (e.g., intensity, duration) and the idea of combining exercise and therapeutic rehabilitation are explored. This exercise-based priming concept has the potential to be applied to many areas such as education, cognitive therapy, and motor rehabilitation.

Keywords: priming, exercise, cognition, motor, rehabilitation

\section{INTRODUCTION}

A lack of cardiovascular fitness has been linked with cognitive dysfunction and learning deficits in various clinical populations (Katz et al., 2012; Alosco et al., 2014); for this reason, recent research efforts have explored the benefits of aerobic exercise on brain health and cognitive functioning with positive results reported for both healthy and neurocognitively impaired individuals (Stoykov et al., 2017). For example, aerobic exercise has been shown to improve memory, processing speed and executive functioning among those with mental deficiencies (Altmann et al., 2016; Zhu et al., 2018), along with facilitating learning in healthy adults (Young et al., 2015; Venckunas et al., 2016; Stern et al., 2019) and adolescents (Berse et al., 2015).

While it appears that aerobic exercise enhances cognitive and motor abilities in humans, it is less well known if gains associated with exercise transfer into improved learning of skills (both motor and cognitive) and learning that many rehabilitation therapies rely heavily upon (e.g., stroke neurorehabilitation, cognitive behavioral therapy). Improved skill learning, such as coping in cognitive behavioral therapy or muscle coordination in physical therapy would ultimately lead to better clinical outcomes. Aerobic exercise may facilitate improvements in treatment outcomes (e.g., abstinence, anxiety, depression, motor skill development) through neural stimulation and plasticity 
promoting mechanisms (e.g., increased blood flow and oxygenation). Cognitive performance components including information processing and memory may be of particular importance for skill acquisition in various rehabilitation programs (Taubert et al., 2015). Therefore, brain activation through aerobic exercise may lead to downstream retention (i.e., memory recall) of cognitive and motor skills taught during therapy or sport coaching. This would be beneficial for those with mood disorders, substance abuse disorders, stroke patients, athletes, and those with neuromuscular injuries. This concept has been defined as "exercise priming" and involves acute exercise stimulation prior to, or after one's engagement in therapy or motor skill training (Charalambous et al., 2018). In practice, performing a brief bout of aerobic exercise prior to cognitive or physical therapy, or before a practice session may lead to improvements in therapeutic or practical outcomes.

Efforts to understand the neuropsychological mechanisms of exercise priming is of vital importance to establish exercise as an adjunct treatment for various therapeutic or learning outcomes. Here, we focus on acute aerobic exercise and its priming effects on cognitive function, learning, and motor skill acquisition. For review papers on the benefits of aerobic, resistance, and combined aerobic and resistance exercise on cognitive performance, please see the reviews of Brunt et al. (2019), Landrigan et al. (2019), Smith et al. (2010), Wilke et al. (2019), and Zheng et al. (2016).

\section{NEUROPSYCHOLOGICAL MECHANISMS OF AEROBIC EXERCISE PRIMING}

\section{Brain Blood Flow and Oxygenation}

Global brain blood flow remains relatively constant during acute aerobic exercise; although, there may be a shift in resources (i.e., oxygen consumption) from areas required for cognitive function to areas required for motor control and maintenance of vital function (e.g., blood pressure and thermoregulation) (Ide and Secher, 2000; Dietrich and Sparling, 2004). In specific cortical regions, blood flow and oxygenation (i.e., activation) are influenced by the intensity of the exercise bout. For example, activation in the prefrontal cortex (PFC), measured by brain oxygenation, increased during submaximal aerobic exercise (up to $80 \%$ of peak ability) but then decreased when intensity reached very hard or maximal effort (Rooks et al., 2010).

Upon cessation of low-moderate intensity aerobic exercise, cerebral oxygenation remains elevated for up to $30 \mathrm{~min}$ (Faulkner et al., 2016; Stavres et al., 2017; Tsubaki et al., 2018). Performing a cognitive task after exercise is potentially ideal as it takes advantage of the heightened cortical activity during recovery. Twenty to $30 \mathrm{~min}$ of moderate intensity (50-60\% $\quad \mathrm{VO}_{2 \max }$ ) cycling increased post-exercise cortical oxygenation (i.e., activation), which aligned with improvements in post-exercise executive function performance (Stroop task) (Yanagisawa et al., 2010; Stavres et al., 2017; Tsubaki et al., 2018). Increased PFC oxygenation may be indicative of higher cortical activity and, therefore, greater mental effort leading to improved cognitive processes such as working memory and attention (Herold et al., 2018). However, a negative association between
left-PFC activation and processing speed has been recently reported among middle aged adults after acute bouts of both low and high intensity aerobic exercise, along with yoga (Moriarty et al., 2019). This acute response may be interpreted as increased neural efficiency, more specifically defined as reduced mental input (lower PFC activation) for mental processing (Causse et al., 2017). The differing results might be in response to the cortical region monitored and the cognitive task performed. For example, neural efficiency improvements for processing speed indicates less PFC neural input required to process information (Rypma et al., 2006). Higher PFC activation during executive function tasks is logical because these measures require memory and attention (Albinet et al., 2014).

In summary, evidence suggests that cerebral blood flow rises during low- to moderate-intensity exercise, and translates to post-exercise alterations in PFC activity, which lead to improvements in various cognitive domains (e.g., executive function and processing speed).

\section{Plasticity and Neurotrophic Factors}

Acute exercise can also enhance the immediate induction of markers of brain plasticity. More specifically, a single 20-30-min bout of moderate and high intensity aerobic exercise led to a transient decrease in short-interval intracortical inhibition and M1 excitability, which are indicators of plasticity in the motor cortex (Smith et al., 2014; Mang et al., 2016a; Singh et al., 2016; Neva et al., 2017). Exercise-induced plasticity has been linked to improvements in cognitive function, such as processing speed (Singh et al., 2016). The plasticity mechanism may be through exercise-induced release of neurotrophins ( $\mathrm{McD}$ Donnell et al., 2013). For the purpose of this review we have decided to focus on two neurotrophins, vascular endothelial growth factor (VEGF) and brain-derived neurotrophic factor (BDNF), both of which have been shown to be upregulated after various types of physical exercise (Maass et al., 2016; Heisz et al., 2017).

Vascular endothelial growth factor is a well-known growth factor and an important signaling molecule involved in angiogenesis and vasculogenesis (Amaral et al., 2001; Lee and Son, 2009). Interestingly, VEGF-A, which is a gene from the VEGF family, increased following either high-intensity exercise or a lactate injection among C57BL/6 mice (Lezi et al., 2013). Gustafsson et al. (1999) also reported that a single bout of dynamic exercise increased VEGF and that there was a graded VEGF response directly related to the metabolic stress of exercise in humans. Therefore, it has been proposed that the VEGF response is from lactate production during exercise. It has been suggested that activation of the lactate receptor (HCAR1) in the brain enhances the effect of VEGF-A and brain angiogenesis, thereby providing a link between aerobic exercise and brain nourishment (Morland et al., 2017). Lactate has also been linked with promoting the expression of plasticity genes (including BDNF) and being required for long-term memory formation and processing (Newman et al., 2011; Schiffer et al., 2011; Suzuki et al., 2011; Yang et al., 2014). Moreover, intravenous infusion of $100 \mathrm{mM}$ L-lactate has been shown to ameliorate cognitive impairment in rats after traumatic brain injury (Holloway et al., 2007). Since brain dysfunctions are associated with 
hypoperfusion and vascular complications, lactate release as a result of exercise may facilitate VEGF expression and act as a potential mechanism for treatment against cognitive decline and other brain conditions.

In addition, BDNF is emerging as a key mediator of synaptic plasticity in the central computational hub for memory processing (i.e., the hippocampus), and is thought to be modulated by insulin growth factor-1 (IGF-1) (Ding et al., 2006). At the cellular level, increases in BDNF may be the link between exercise and learning; however, BDNF induction in response to acute exercise is mixed, and may be influenced by the intensity of exercise, along with the level of cognitive impairment among research participants (Charalambous et al., 2018). BDNF is thought to regulate synaptic proteins (e.g., synapsin I and synaptophysin) within the hippocampus thereby improving axonal branching and allowing for an increased effectiveness in synaptic transmission (Danzer et al., 2002; Vaynman et al., 2004). Korte et al. (1995) blocked the expression of BDNF in mice and found them to have a significantly reduced long-term potentiation (a measure of synaptic plasticity). Heldt et al. (2007) found that BDNF deletion from the hippocampus impaired novel object recognition and spatial learning in mice. Importantly, these impairments are reversed when exogenous BDNF is given to a BDNF-deficient animal (Patterson et al., 1996), further providing support for the importance of this neurotrophic factor in neural and cognitive function (Cotman and Berchtold, 2002).

In humans, serum BDNF is commonly measured as an indirect indicator of neurogenesis. This is based on evidence that BDNF produced in the brain accounts for $70-80 \%$ of circulating BDNF in response to aerobic exercise in humans (Rasmussen et al., 2009). The increase in serum BDNF has been reported in response to acute bouts of aerobic exercise and also linked with better hippocampal function (Griffin et al., 2011). The magnitude of the increase in serum BDNF in humans may be exercise intensity dependent. Ferris et al. (2007) reported a 13 and $30 \%$ increase in serum BDNF following cycling above ventilatory threshold or graded exercise test to volitional fatigue, respectively. More recently, Ross et al. (2019) reported a direct linear relationship between exercise intensity and post-exercise serum BDNF among both healthy and moderately depressed individuals. The intensity-dependent increase in serum BDNF has also been positively associated with improved prefrontal cognitive functioning in humans (Hwang et al., 2016). Conversely, Tsai et al. (2014, 2016, 2018) found no relationship between increased BDNF concentrations and improved cognitive performance following 30-min of moderateintensity exercise in both healthy adults and older adults with mild cognitive impairment. Accordingly, these results purport that the circulating BDNF response to exercise is not linked to post-exercise cognitive performance. The transient increase in BDNF after acute exercise may explain the lack of correlations with cognitive performance. Therefore, it is possible that the post-exercise timing of the serum BDNF measurements align with the cognitive testing influenced outcomes (Tsai et al., 2018). Alternately, non-BDNF mechanisms (neurotrophins, arousal, hormones) could be responsible for the cognitive changes after exercise.
While conflicting, these data indicate that acute aerobic exercise can improve cognitive function concomitant with increased serum BDNF concentrations, thereby suggesting a functional role for this neurotrophic factor in acute exerciseinduced cognitive enhancement in humans. However, the BDNF changes may not be the only potential factor that drives improvements in cognitive performance after aerobic exercise.

In summary, motor plasticity in response to acute aerobic exercise may induce the release of neurotrophins, VEGF and BDNF, which, are linked to cognitive performance improvements.

\section{Neuroendocrine and Myokines}

Catecholamines, such as norepinephrine (NE) and dopamine (DA) have been attributed to the cognitive benefits of acute exercise, which has been defined as the "catecholamine hypothesis" (McMorris, 2016). According to this model, acute short duration, moderate intensity exercise stimulates NE synthesis in the PFC region leading to increased arousal and attention. Conversely, during long duration, high intensity exercise, a larger release of $\mathrm{NE}$, along with DA dampen neuronal activity causing a decline in executive functioning (McMorris, 2016). However, NE released during long duration, moderate intensity aerobic exercise has been shown to facilitate the synthesis of brain BDNF (McMorris, 2016). Thus, catecholamine release supports brain function after moderate intensity exercise, regardless of duration.

Acute aerobic exercise at approximately 60\% intensity, or higher stimulates the hypothalamic-pituitary-adrenal (HPA) axis and increases the secretion of cortisol, which peaks around $30 \mathrm{~min}$ post exercise and remains elevated for $2 \mathrm{~h}$ (Duclos et al., 1998; Hill et al., 2008). Evidence suggests that the cortisol released improves learning and memory by interacting with glucocorticoid and mineralocorticoid receptors located in the hippocampus, amygdala, and PFC regions of the brain (Heffelfinger and Newcomer, 2001; Yuen et al., 2009). A positive correlation was detected between the increase in cortisol release after exercise and vocabulary retention among healthy adults (Hötting et al., 2016). Interestingly, cortisol released in response to a psychosocial stress task resulted in an impaired retrieval of words (Tollenaar et al., 2008). The differing memory responses may be due to the higher cortisol released during the psychological stress compared to physical stress. Large increases in cortisol impairs memory, thus indicating a possible threshold for cortisol mediation of cognitive function (Dominique et al., 2000; Basso and Suzuki, 2017).

Myokines, which are released from skeletal muscle, may also help to explain the cognitive and motor functioning benefits of acute exercise (Kim et al., 2019). Of note, irisin has been shown to attenuate brain damage incurred during various types of cerebral insult (ischemia, stroke) (Asadi et al., 2018). Irisin functions via activation of Akt and ERK1/2 pro-survival signaling pathways thereby reducing ischemia-induced neuronal injury ( $\mathrm{Li}$ et al., 2017). In addition, aerobic exercise increased the expression of FNDC5 (a membrane protein that is cleaved and secreted as irisin), which led to upregulation of BDNF in the hippocampus, thus demonstrating a link between exercise-induced irisin and 
neurogenesis (Wrann et al., 2013). Cathepsin B is another recently identified myokine that is important for neural plasticity and cognitive function (Moon et al., 2016). Aerobic exercise has been shown to increase cathepsin B plasma levels in mice, monkeys, and humans (Moon et al., 2016). In humans, changes in cathepsin B positively correlated with fitness and memory (Moon et al., 2016). Though, to date, little research has been conducted on myokines' effect on the brain; however, both irisin and cathepsin B may play important roles in the beneficial effects of exercise on brain health and function.

In summary, acute aerobic exercise appears to promote cognitive gains which may, in part, be mediated through cerebral blood flow and cortical activation, growth and neurotrophic factors, as well as hormones and myokines. For this reason, researchers are beginning to examine the role of exercise as a cognition-stimulating mechanism to improve cognitive performance and enhance motor skill acquisition in both healthy and clinical populations (Lefferts et al., 2019).

\section{ACUTE AEROBIC EXERCISE AS A PRIMING TECHNIQUE FOR COGNITIVE IMPROVEMENTS AND MOTOR SKILL ACQUISITION}

"Exercise priming" refers to a non-conscious process that promotes cognitive or motor skill related learning, whereby performing an acute exercise bout alters the response of another stimulus (Stoykov et al., 2017). Priming relies on the transient cognitive benefits of acute aerobic exercise and, when strategically performed before or after a task (either motor or cognitive), may improve learning outcomes. In humans, commonly studied cognitive domains include information processing, reaction time, memory, executive functioning, and attention (Chang et al., 2012). Typical study protocols include a baseline measurement of the specific cognitive domain(s) or motor task of interest followed by an acute aerobic exercise session and then retesting of the cognitive domain(s) or motor task. Acute bouts of aerobic exercise ranging from 10 to $30 \mathrm{~min}$ at an intensity of $40-100 \%$ of maximal intensity have stimulated improvements in various cognitive domains and motor tasks.

For example, young adults demonstrated improved reaction times after performing acute moderate-intensity (50-70\% of maximal heart rate) exercise for 20-30 min (Sibley et al., 2006; Harveson et al., 2016; Wang et al., 2019). Cognitive improvements have also been reported among clinical populations (e.g., patients with Multiple Sclerosis, depression, breast cancer) after acute bouts of moderate-intensity activity (Vasques et al., 2011; Sandroff et al., 2015; Salerno et al., 2019). Recently, Lefferts et al. (2019) found that a 30-min bout of moderate-intensity cycling decreased reaction time in the Eriksen Flanker task and in a memory recognition task in middle-aged individuals with hypertension. Support for the "priming" effect on behavioral performance has also been illustrated in post-stroke patients following an acute 15-min bout of cycling as reported by an improvement in the behavioral performance of a working memory task compared with the non-exercise control condition (Moriya et al., 2016). In addition, acute short duration (15-20 min) exercise has also been shown to improve motor skill learning and performance (Roig et al., 2012; Mang et al., 2014, 2016b; Skriver et al., 2014; Stavrinos and Coxon, 2017; Dal Maso et al., 2018). For example, Mang et al. (2016b) and Roig et al. (2012) found that 20 min of high-intensity interval cycling (3-min at $90 \% \mathrm{VO}_{2 \text { peak }}$ alternating with 3-min recovery) performed prior to motor skill practice, improved memory recall of the skill in response to a 24 -h delayed retention test. In addition, several research groups have reported improved motor skill retention when exercise is performed after practicing the motor skill (Roig et al., 2012; Stavrinos and Coxon, 2017; Dal Maso et al., 2018). The authors suggested the improvement occurred as a result of an increased rate of motor memory retrieval and learning (Mang et al., 2014, 2016b). More recent evidence has also found that the effects of performing exercise after practicing a motor task are beneficial for retention of that particular task (Stavrinos and Coxon, 2017; Dal Maso et al., 2018). Conversely, no improvements in cognitive and motor function after acute exercise has also been reported (Thomas et al., 2017; Moriarty et al., 2019). The null findings have been attributed to the exercise stimulus administered and also to the timing of cognitive or motor testing (Chang et al., 2012; Thomas et al., 2016). A greater stimulus (more intense exercise), along with administering testing at least 20 min post-exercise appear to influence cognitive and motor results (Chang et al., 2012). Other reported factors, include cognitive or motor construct measured, and the health status of the participants (Chang et al., 2012). In addition, previous research in both adolescent and adult populations have argued that those with higher fitness levels demonstrate greater neural efficiency, and in turn, greater improvements in cognitive performance (Stroth et al., 2009; Renaud et al., 2010; Chan et al., 2011; Hogan et al., 2013). In summary, a fair amount of evidence suggests that acute exercise leads to cognitive improvements and motor skill acquisition among young and older adults, along with clinical populations.

The evidence presented above suggests that acute exercise positioned proximal to (before or after) cognitive or motor tasks leads to performance improvements (see Figure 1). Based on these data, exercise may serve as an adjunctive therapy to cognitive or motor (i.e., physical) therapies. Examples include an aerobic exercise program designed to operate alongside cognitive behavioral therapy (CBT) for treatment of substance use disorder or severe depression or an aerobic exercise program in conjunction with physical therapy for stroke patients. Early onset of cognitive deficits can signal brain and behavioral disorders such as schizophrenia and Alzheimer's disease (Uhlhaas and Singer, 2006). The cognitive or motor function improvements induced by exercise may "prime" the patient to more fully engage in and benefit from therapeutic tasks for various treatments. Limited efforts have been made to partner exercise with therapy; whereas, the bulk of research has compared exercise alone to therapy (Stathopoulou et al., 2006; Zschucke et al., 2013). We argue that exercise cannot replace cognitive or motor therapy but may be able to enhance the benefits. For example, exercise combined with cognitive therapy for the 


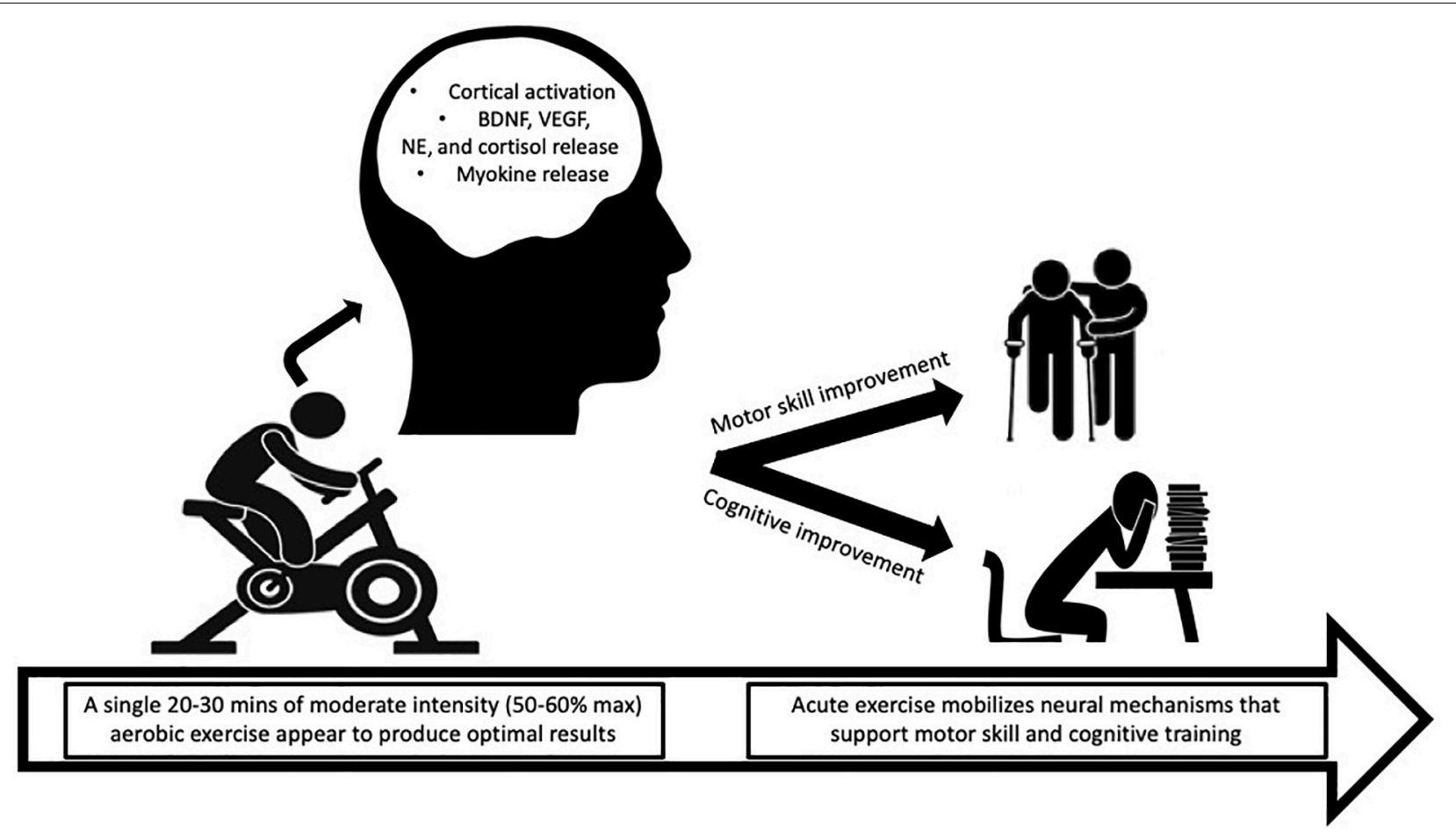

FIGURE 1 | An acute 20-30-min bout of moderate-intensity aerobic exercise may mobilize neural mechanisms that help prime the brain for cognitive and motor performance. BDNF, brain-derived neurotrophic factor; VEGF, vascular endothelial growth factor; NE, norepinephrine.

treatment of schizophrenia improved symptoms and functional outcomes compared to cognitive therapy alone (Malchow et al., 2016; Nuechterlein et al., 2016). In addition, stimulant users report more abstinent days when they participate in exercise combined with therapy compared to therapy partnered with health education (Trivedi et al., 2017).

In summary, both acute and chronic exercise training studies have provided initial evidence that aerobic exercise improves cognitive and motor function in cognitively impaired individuals. Specifically, the cognitive benefits associated with such exercise interventions have been shown to extend beyond improving mobility of limbs to also improving sensorimotor learning and performance in cognitive tasks. Therefore, aerobic exercise in combination with therapeutic recovery and motor control rehabilitation techniques may ultimately augment functional outcomes through cognitive effects. As the number of individuals with cognitive and motor impairments continue to rise, it is imperative that more research is conducted in this area to define the specific parameters (e.g., intensity, duration) and combinations (e.g., exercise and therapy) of such chronic exercise interventions.

\section{CONCLUSION}

Aerobic exercise, both acute and chronic, has the ability to prime the brain for both cognitive and motor task performance. These findings provide a stable groundwork for designing and prescribing acute aerobic exercise in future research studies examining the effects of exercise on cognitive performance and motor skill acquisition. Applying this priming idea to education, rehabilitation, and therapy has the potential for improved cognitive and motor performance and may form an important component of improvements in these fields. Finally, the combination of exercise and various forms of therapeutic rehabilitation may enhance the functional outcomes and quality of life for individuals with cognitive or motor impairments and perhaps be the way of the future with further in-depth research and knowledge of mechanisms.

\section{AUTHOR CONTRIBUTIONS}

TM contributed to writing the first draft of the manuscript, conception and design of the manuscript, and revising the manuscript critically. CM, LK, AG, and NB contributed to revising the manuscript critically for important intellectual content, initiating the idea, and monitoring progress. MZ contributed to writing the manuscript, conception and design of the manuscript, and monitoring progress. All authors contributed to the manuscript revision, and read and approved the submitted version.

\section{FUNDING}

This work was supported by the University of Northern Iowa, The University of New Mexico, and Central Michigan University. 


\section{REFERENCES}

Albinet, C. T., Mandrick, K., Bernard, P. L., Perrey, S., and Blain, H. (2014). Improved cerebral oxygenation response and executive performance as a function of cardiorespiratory fitness in older women: a fNIRS study. Front. Aging Neurosci. 6:272. doi: 10.3389/fnagi.2014.00272

Alosco, M. L., Spitznagel, M. B., Cohen, R., Raz, N., Sweet, L. H., Josephson, R., et al. (2014). Decreased physical activity predicts cognitive dysfunction and reduced cerebral blood flow in heart failure. J. Neurol. Sci. 339, 169-175. doi: 10.1016/j.jns.2014.02.008

Altmann, L. J., Stegemöller, E., Hazamy, A. A., Wilson, J. P., Bowers, D., Okun, M. S., et al. (2016). Aerobic exercise improves mood, cognition, and language function in parkinson's disease: results of a controlled study. J. Int. Neuropsychol. Soc. 22, 878-889. doi: 10.1017/S135561771600076X

Amaral, S. L., Papanek, P. E., and Greene, A. S. (2001). Angiotensin II and VEGF are involved in angiogenesis induced by short-term exercise training. Am. J. Physiol. Heart Circ. Physiol. 281, H1163-H1169. doi: 10.1152/ajpheart.2001.281. 3.H1163

Asadi, Y., Gorjipour, F., Behrouzifar, S., and Vakili, A. (2018). Irisin peptide protects brain against ischemic injury through reducing apoptosis and enhancing BDNF in a rodent model of stroke. Neurochem. Res. 43, 1549-1560. doi: 10.1007/s11064-018-2569-9

Basso, J. C., and Suzuki, W. A. (2017). The effects of acute exercise on mood, cognition, neurophysiology, and neurochemical pathways: a review. Brain Plast. 2, 127-152. doi: 10.3233/BPL-160040

Berse, T., Rolfes, K., Barenberg, J., Dutke, S., Kuhlenbäumer, G., Völker, K., et al. (2015). Acute physical exercise improves shifting in adolescents at school: evidence for a dopaminergic contribution. Front. Behav. Neurosci. 9:196. doi: 10.3389/fnbeh.2015.00196

Brunt, A., Albines, D., and Hopkins-Rosseel, D. (2019). The effectiveness of exercise on cognitive performance in individuals with known vascular disease: a systematic review. J. Clin. Med. 8:294. doi: 10.3390/jcm8030294

Causse, M., Chua, Z., Peysakhovich, V., Del Campo, N., and Matton, N. (2017). Mental workload and neural efficiency quantified in the prefrontal cortex using fNIRS. Sci. Rep. 7:5222. doi: 10.1038/s41598-017-05378-x

Chan, J. S., Wong, A. C., Liu, Y., Yu, J., and Yan, J. H. (2011). Fencing expertise and physical fitness enhance action inhibition. Psychol. Sport Exerc. 12, 509-514. doi: 10.1016/j.psychsport.2011.04.006

Chang, Y.-K., Labban, J. D., Gapin, J. I., and Etnier, J. L. (2012). The effects of acute exercise on cognitive performance: a meta-analysis. Brain Res. 1453, 87-101. doi: 10.1016/j.brainres.2012.02.068

Charalambous, C. C., Alcantara, C. C., French, M. A., Li, X., Matt, K. S., Kim, H. E., et al. (2018). A single exercise bout and locomotor learning after stroke: physiological, behavioural, and computational outcomes. J. Physiol. 596, 1999-2016. doi: 10.1113/JP275881

Cotman, C. W., and Berchtold, N. C. (2002). Exercise: a behavioral intervention to enhance brain health and plasticity. Trends Neurosci. 25, 295-301. doi: 10.1016/ S0166-2236(02)02143-4

Dal Maso, F., Desormeau, B., Boudrias, M. H., and Roig, M. (2018). Acute cardiovascular exercise promotes functional changes in cortico-motor networks during the early stages of motor memory consolidation. Neuroimage 174, 380-392. doi: 10.1016/j.neuroimage.2018.03.029

Danzer, S. C., Crooks, K. R., Lo, D. C., and McNamara, J. O. (2002). Increased expression of brain-derived neurotrophic factor induces formation of basal dendrites and axonal branching in dentate granule cells in hippocampal explant cultures. J. Neurosci. 22, 9754-9763. doi: 10.1523/JNEUROSCI.22-22-09754. 2002

Dietrich, A., and Sparling, P. B. (2004). Endurance exercise selectively impairs prefrontal-dependent cognition. Brain Cogn. 55, 516-524. doi: 10.1016/j.bandc. 2004.03.002

Ding, Q., Vaynman, S., Akhavan, M., Ying, Z., and Gomez-Pinilla, F. (2006). Insulin-like growth factor I interfaces with brain-derived neurotrophic factormediated synaptic plasticity to modulate aspects of exercise-induced cognitive function. Neuroscience 140, 823-833. doi: 10.1016/j.neuroscience.2006.02.084

Dominique, J.-F., Roozendaal, B., Nitsch, R. M., McGaugh, J. L., and Hock, C. (2000). Acute cortisone administration impairs retrieval of long-term declarative memory in humans. Nat. Neurosci. 3:313. doi: 10.1038/73873
Duclos, M., Corcuff, J., Arsac, L., Moreau-Gaudry, F., Rashedi, M., Roger, P., et al. (1998). Corticotroph axis sensitivity after exercise in endurance-trained athletes. Clin. Endocrinol. 48, 493-501. doi: 10.1046/j.1365-2265.1998.00334.x

Faulkner, J., Lambrick, D., Kaufmann, S., and Stoner, L. (2016). Effects of upright and recumbent cycling on executive function and prefrontal cortex oxygenation in young healthy men. J. Phys. Activity Health 13, 882-887. doi: 10.1123/jpah. 2015-0454

Ferris, L. T., Williams, J. S., and Shen, C.-L. (2007). The effect of acute exercise on serum brain-derived neurotrophic factor levels and cognitive function. Med. Sci. Sports Exerc. 39, 728-734. doi: 10.1249/mss.0b013e31802f04c7

Griffin, ÉW., Mullally, S., Foley, C., Warmington, S. A., O’Mara, S. M., and Kelly, ÁM. (2011). Aerobic exercise improves hippocampal function and increases BDNF in the serum of young adult males. Physiol. Behav. 104, 934-941. doi: 10.1016/j.physbeh.2011.06.005

Gustafsson, T., Puntschart, A., Kaijser, L., Jansson, E., and Sundberg, C. J. (1999). Exercise-induced expression of angiogenesis-related transcription and growth factors in human skeletal muscle. Am. J. Physiol. Heart Circ. Physiol. 276, H679-H685. doi: 10.1152/ajpheart.1999.276.2.H679

Harveson, A. T., Hannon, J. C., Brusseau, T. A., Podlog, L., Papadopoulos, C., Durrant, L. H., et al. (2016). Acute effects of 30 minutes resistance and aerobic exercise on cognition in a high school sample. Res. Q. Exerc. Sport 87, 214-220. doi: 10.1080/02701367.2016.1146943

Heffelfinger, A. K., and Newcomer, J. W. (2001). Glucocorticoid effects on memory function over the human life span. Dev. Psychopathol. 13, 491-513. doi: 10.1017/ S0954579401003054

Heisz, J. J., Clark, I. B., Bonin, K., Paolucci, E. M., Michalski, B., Becker, S., et al. (2017). The effects of physical exercise and cognitive training on memory and neurotrophic factors. J. Cogn. Neurosci. 29, 1895-1907. doi: 10.1162/jocn_a_ 01164

Heldt, S., Stanek, L., Chhatwal, J., and Ressler, K. (2007). Hippocampus-specific deletion of BDNF in adult mice impairs spatial memory and extinction of aversive memories. Mol. Psychiatry 12:656. doi: 10.1038/sj.mp.4001957

Herold, F., Wiegel, P., Scholkmann, F., and Mueller, N. G. (2018). Applications of functional near-infrared spectroscopy (fNIRS) neuroimaging in ExerciseCognition science: a systematic. Methodology-focused review. J. Clin. Med. 7:466. doi: $10.3390 /$ jcm7120466

Hill, E., Zack, E., Battaglini, C., Viru, M., Viru, A., and Hackney, A. (2008). Exercise and circulating cortisol levels: the intensity threshold effect. J. Endocrinol. Invest. 31, 587-591. doi: 10.1007/bf03345606

Hogan, M., Kiefer, M., Kubesch, S., Collins, P., Kilmartin, L., and Brosnan, M. (2013). The interactive effects of physical fitness and acute aerobic exercise on electrophysiological coherence and cognitive performance in adolescents. Exp. Brain Res. 229, 85-96. doi: 10.1007/s00221-013-3595-0

Holloway, R., Zhou, Z., Harvey, H., Levasseur, J., Rice, A., Sun, D., et al. (2007). Effect of lactate therapy upon cognitive deficits after traumatic brain injury in the rat. Acta Neurochirurgica 149, 919-927. doi: 10.1007/s00701-007$1241-\mathrm{y}$

Hötting, K., Schickert, N., Kaiser, J., Röder, B., and Schmidt-Kassow, M. (2016). The effects of acute physical exercise on memory, peripheral BDNF, and cortisol in young adults. Neural Plast. 2016:6860573. doi: 10.1155/2016/6860573

Hwang, J., Brothers, R. M., Castelli, D. M., Glowacki, E. M., Chen, Y. T., Salinas, M. M., et al. (2016). Acute high-intensity exercise-induced cognitive enhancement and brain-derived neurotrophic factor in young, healthy adults. Neurosci. Lett. 630, 247-253. doi: 10.1016/j.neulet.2016.07.033

Ide, K., and Secher, N. H. (2000). Cerebral blood flow and metabolism during exercise. Prog. Neurobiol. 61, 397-414. doi: 10.1016/S0301-0082(99)00057-X

Katz, P., Julian, L., Tonner, M. C., Yazdany, J., Trupin, L., Yelin, E., et al. (2012). Physical activity, obesity, and cognitive impairment among women with systemic lupus erythematosus. Arthr. Care Res. 64, 502-510. doi: 10.1002/acr. 21587

Kim, S., Choi, J. Y., Moon, S., Park, D. H., Kwak, H. B., and Kang, J. H. (2019). Roles of myokines in exercise-induced improvement of neuropsychiatric function. Pflugers Arch. 471, 491-505. doi: 10.1007/s00424-019-02253-8

Korte, M., Carroll, P., Wolf, E., Brem, G., Thoenen, H., and Bonhoeffer, T. (1995). Hippocampal long-term potentiation is impaired in mice lacking brain-derived neurotrophic factor. Proc. Natl. Acad. Sci. U.S.A. 92, 8856-8860. doi: 10.1073/ pnas.92.19.8856 
Landrigan, J.-F., Bell, T., Crowe, M., Clay, O. J., and Mirman, D. (2019). Lifting cognition: a meta-analysis of effects of resistance exercise on cognition. Psychol. Res. doi: 10.1007/s00426-019-01145-x [Epub ahead of print].

Lee, E.-G., and Son, H. (2009). Adult hippocampal neurogenesis and related neurotrophic factors. BMB Rep. 42, 239-244. doi: 10.5483/BMBRep.2009.42. 5.239

Lefferts, W. K., DeBlois, J. P., White, C. N., and Heffernan, K. S. (2019). Effects of acute aerobic exercise on cognition and constructs of decision-making in adults with and without hypertension. Front. Aging Neurosci. 11:41. doi: 10.3389/fnagi. 2019.00041

Lezi, E., Lu, J., Selfridge, J. E., Burns, J. M., and Swerdlow, R. H. (2013). Lactate administration reproduces specific brain and liver exercise-related changes. J. Neurochem. 127, 91-100. doi: 10.1111/jnc. 12394

Li, D.-J., Li, Y.-H., Yuan, H.-B., Qu, L.-F., and Wang, P. (2017). The novel exerciseinduced hormone irisin protects against neuronal injury via activation of the Akt and ERK1/2 signaling pathways and contributes to the neuroprotection of physical exercise in cerebral ischemia. Metabolism 68, 31-42. doi: 10.1016/ j.metabol.2016.12.003

Maass, A., Düzel, S., Brigadski, T., Goerke, M., Becke, A., Sobieray, U., et al. (2016). Relationships of peripheral IGF-1, VEGF and BDNF levels to exercise-related changes in memory, hippocampal perfusion and volumes in older adults. Neuroimage 131, 142-154. doi: 10.1016/j.neuroimage.2015.10.084

Malchow, B., Keeser, D., Keller, K., Hasan, A., Rauchmann, B.-S., Kimura, H., et al. (2016). Effects of endurance training on brain structures in chronic schizophrenia patients and healthy controls. Schizophrenia Res. 173, 182-191. doi: 10.1016/j.schres.2015.01.005

Mang, C. S., Brown, K. E., Neva, J. L., Snow, N. J., Campbell, K. L., and Boyd, L. A. (2016a). Promoting motor cortical plasticity with acute aerobic exercise: a role for cerebellar circuits. Neural Plast. 2016:6797928. doi: 10.1155/2016/6797928

Mang, C. S., Snow, N. J., Campbell, K. L., Ross, C. J., and Boyd, L. A. (2014). A single bout of high-intensity aerobic exercise facilitates response to paired associative stimulation and promotes sequence-specific implicit motor learning. J. Appl. Physiol. 117, 1325-1336. doi: 10.1152/japplphysiol.00498.2014

Mang, C. S., Snow, N. J., Wadden, K. P., Campbell, K. L., and Boyd, L. A. (2016b). High-intensity aerobic exercise enhances motor memory retrieval. Med. Sci. Sports Exerc. 48, 2477-2486. doi: 10.1249/MSS.0000000000001040

McDonnell, M. N., Buckley, J. D., Opie, G. M., Ridding, M. C., and Semmler, J. G. A. (2013). single bout of aerobic exercise promotes motor cortical neuroplasticity. J. Appl. Physiol. 114, 1174-1182. doi: 10.1152/japplphysiol.01378.2012

McMorris, T. (2016). Developing the catecholamines hypothesis for the acute exercise-cognition interaction in humans: lessons from animal studies. Physiol. Behav. 165, 291-299. doi: 10.1016/j.physbeh.2016.08.011

Moon, H. Y., Becke, A., Berron, D., Becker, B., Sah, N., Benoni, G., et al. (2016). Running-induced systemic cathepsin B secretion is associated with memory function. Cell Metab. 24, 332-340. doi: 10.1016/j.cmet.2016.05.025

Moriarty, T., Bourbeau, K., Bellovary, B., and Zuhl, M. N. (2019). Exercise intensity influences prefrontal cortex oxygenation during cognitive testing. Behav. Sci. 9:E83. doi: 10.3390/bs9080083

Moriya, M., Aoki, C., and Sakatani, K. (2016). Effects of Physical Exercise on Working Memory and Prefrontal Cortex Function in Post-Stroke Patients. Oxygen Transport to Tissue XXXVIII. Berlin: Springer, 203-208. doi: 10.1007/ 978-3-319-38810-6_27

Morland, C., Andersson, K. A., Haugen, ØP., Hadzic, A., Kleppa, L., Gille, A., et al. (2017). Exercise induces cerebral VEGF and angiogenesis via the lactate receptor HCAR1. Nat. Commun. 8:15557. doi: 10.1038/ncomms15557

Neva, J., Brown, K., Mang, C., Francisco, B., and Boyd, L. (2017). An acute bout of exercise modulates both intracortical and interhemispheric excitability. Eur. J. Neurosci. 45, 1343-1355. doi: 10.1111/ejn.13569

Newman, L. A., Korol, D. L., and Gold, P. E. (2011). Lactate produced by glycogenolysis in astrocytes regulates memory processing. PLoS One 6:e28427. doi: 10.1371/journal.pone.0028427

Nuechterlein, K. H., Ventura, J., McEwen, S. C., Gretchen-Doorly, D., Vinogradov, S., and Subotnik, K. L. (2016). Enhancing cognitive training through aerobic exercise after a first schizophrenia episode: theoretical conception and pilot study. Schizophrenia Bull. 42(Suppl._1), S44-S52. doi: 10.1093/schbul/sbw007

Patterson, S. L., Abel, T., Deuel, T. A., Martin, K. C., Rose, J. C., and Kandel, E. R. (1996). Recombinant BDNF rescues deficits in basal synaptic transmission and hippocampal LTP in BDNF knockout mice. Neuron 16, 1137-1145. doi: 10.1016/S0896-6273(00)80140-3
Rasmussen, P., Brassard, P., Adser, H., Pedersen, M. V., Leick, L., Hart, E., et al. (2009). Evidence for a release of brain-derived neurotrophic factor from the brain during exercise. Exp. Physiol. 94, 1062-1069. doi: 10.1113/expphysiol. 2009.048512

Renaud, M., Bherer, L., and Maquestiaux, F. (2010). A high level of physical fitness is associated with more efficient response preparation in older adults. J. Gerontol. Ser B 65, 317-322. doi: 10.1093/geronb/gbq004

Roig, M., Skriver, K., Lundbye-Jensen, J., Kiens, B., and Nielsen, J. B. (2012). A single bout of exercise improves motor memory. PLoS One 7:e44594. doi: 10.1371/journal.pone.0044594

Rooks, C. R., Thom, N. J., McCully, K. K., and Dishman, R. K. (2010). Effects of incremental exercise on cerebral oxygenation measured by near-infrared spectroscopy: a systematic review. Prog. Neurobiol. 92, 134-150. doi: 10.1016/ j.pneurobio.2010.06.002

Ross, R. E., Saladin, M. E., George, M. S., and Gregory, C. M. (2019). High-intensity aerobic exercise acutely increases brain-derived neurotrophic factor. Med. Sci. Sports Exerc. 51, 1698-1709 doi: 10.1249/mss.0000000000001969

Rypma, B., Berger, J. S., Prabhakaran, V., Bly, B. M., Kimberg, D. Y., Biswal, B. B., et al. (2006). Neural correlates of cognitive efficiency. Neuroimage 33, 969-979. doi: 10.1016/j.neuroimage.2006.05.065

Salerno, E. A., Rowland, K., Kramer, A. F., and McAuley, E. (2019). Acute aerobic exercise effects on cognitive function in breast cancer survivors: a randomized crossover trial. BMC Cancer 19:371. doi: 10.1186/s12885-019-5589-1

Sandroff, B. M., Hillman, C. H., Benedict, R. H., and Motl, R. W. (2015). Acute effects of walking, cycling, and yoga exercise on cognition in persons with relapsing-remitting multiple sclerosis without impaired cognitive processing speed. J. Clin. Exp. Neuropsychol. 37, 209-219. doi: 10.1080/13803395.2014. 1001723

Schiffer, T., Schulte, S., Sperlich, B., Achtzehn, S., Fricke, H., and Strüder, H. K. (2011). Lactate infusion at rest increases BDNF blood concentration in humans. Neurosci. Lett. 488, 234-237. doi: 10.1016/j.neulet.2010.11.035

Sibley, B. A., Etnier, J. L., and Le Masurier, G. C. (2006). Effects of an acute bout of exercise on cognitive aspects of Stroop performance. J. Sport Exerc. Psychol. 28, 285-299. doi: 10.1123/jsep.28.3.285

Singh, A. M., Neva, J. L., and Staines, W. R. (2016). Aerobic exercise enhances neural correlates of motor skill learning. Behav. Brain Res. 301, 19-26. doi: 10.1016/j.bbr.2015.12.020

Skriver, K., Roig, M., Lundbye-Jensen, J., Pingel, J., Helge, J. W., Kiens, B., et al. (2014). Acute exercise improves motor memory: exploring potential biomarkers. Neurobiol. Learn. Mem. 116, 46-58. doi: 10.1016/j.nlm.2014. 08.004

Smith, A. E., Goldsworthy, M. R., Garside, T., Wood, F. M., and Ridding, M. C. (2014). The influence of a single bout of aerobic exercise on short-interval intracortical excitability. Exp. Brain Res. 232, 1875-1882. doi: 10.1007/s00221014-3879-z

Smith, P. J., Blumenthal, J. A., Hoffman, B. M., Cooper, H., Strauman, T. A., WelshBohmer, K., et al. (2010). Aerobic exercise and neurocognitive performance: a meta-analytic review of randomized controlled trials. Psychosom. Med. 72:239. doi: 10.1097/PSY.0b013e3181d14633

Stathopoulou, G., Powers, M. B., Berry, A. C., Smits, J. A., and Otto, M. W. (2006). Exercise interventions for mental health: a quantitative and qualitative review. Clin. Psychol. 13, 179-193. doi: 10.1111/j.1468-2850.2006.00021.x

Stavres, J., Gerhart, H. D., Kim, J.-H., Glickman, E. L., and Seo, Y. (2017). Cerebral hemodynamics and executive function during exercise and recovery in normobaric hypoxia. Aerosp. Med. Hum. Perform. 88, 911-917. doi: 10.3357/ AMHP.4830.2017

Stavrinos, E. L., and Coxon, J. P. (2017). High-intensity interval exercise promotes motor cortex disinhibition and early motor skill consolidation. J. Cogn. Neurosci. 29, 593-604. doi: 10.1162/jocn_a_01078

Stern, Y., MacKay-Brandt, A., Lee, S., McKinley, P., McIntyre, K., Razlighi, Q., et al. (2019). Effect of aerobic exercise on cognition in younger adults: a randomized clinical trial. Neurology 92, e905-e916. doi: 10.1212/WNL.0000000000007003

Stoykov, M. E., Corcos, D. M., and Madhavan, S. (2017). Movement-based priming: clinical applications and neural mechanisms. J. Motor Behav. 49, 88-97. doi: 10.1080/00222895.2016.1250716

Stroth, S., Kubesch, S., Dieterle, K., Ruchsow, M., Heim, R., and Kiefer, M. (2009). Physical fitness, but not acute exercise modulates event-related potential indices for executive control in healthy adolescents. Brain Res. 1269, 114-124. doi: 10.1016/j.brainres.2009.02.073 
Suzuki, A., Stern, S. A., Bozdagi, O., Huntley, G. W., Walker, R. H., Magistretti, P. J., et al. (2011). Astrocyte-neuron lactate transport is required for long-term memory formation. Cell 144, 810-823. doi: 10.1016/j.cell.2011.02.018

Taubert, M., Villringer, A., and Lehmann, N. (2015). Endurance exercise as an "endogenous" neuro-enhancement strategy to facilitate motor learning. Front. Hum. Neurosci. 9:692. doi: 10.3389/fnhum.2015.00692

Thomas, R., Flindtgaard, M., Skriver, K., Geertsen, S. S., Christiansen, L., Korsgaard Johnsen, L., et al. (2017). Acute exercise and motor memory consolidation: does exercise type play a role? Scand. J. Med. Sci. Sports 27, 1523-1532. doi: 10.1111/sms.12791

Thomas, R., Johnsen, L. K., Geertsen, S. S., Christiansen, L., Ritz, C., Roig, M., et al. (2016). Acute exercise and motor memory consolidation: the role of exercise intensity. PLoS One 11:e0159589. doi: 10.1371/journal.pone.0159589

Tollenaar, M. S., Elzinga, B. M., Spinhoven, P., and Everaerd, W. A. (2008). The effects of cortisol increase on long-term memory retrieval during and after acute psychosocial stress. Acta Psychol. 127, 542-552. doi: 10.1016/j.actpsy.2007. 10.007

Trivedi, M. H., Greer, T. L., Rethorst, C. D., Carmody, T., Grannemann, B. D., Walker, R., et al. (2017). Randomized trial comparing exercise to health education for stimulant use disorder: results from STimulant Reduction Intervention using Dosed Exercise (CTN-0037; STRIDE). J. Clin Psychiatry 78:1075. doi: 10.4088/JCP.15m10591

Tsai, C. L., Pan, C. Y., Chen, F. C., Wang, C. H., and Chou, F. Y. (2016). Effects of acute aerobic exercise on a task-switching protocol and brain-derived neurotrophic factor concentrations in young adults with different levels of cardiorespiratory fitness. Exp. Physiol. 101, 836-850. doi: 10.1113/EP085682

Tsai, C.-L., Chen, F.-C., Pan, C.-Y., Wang, C.-H., Huang, T.-H., and Chen, T.-C. (2014). Impact of acute aerobic exercise and cardiorespiratory fitness on visuospatial attention performance and serum BDNF levels. Psychoneuroendocrinology 41, 121-131. doi: 10.1016/j.psyneuen.2013.12.014

Tsai, C.-L., Ukropec, J., Ukropcová, B., and Pai, M.-C. (2018). An acute bout of aerobic or strength exercise specifically modifies circulating exerkine levels and neurocognitive functions in elderly individuals with mild cognitive impairment. Neuroimage 17, 272-284. doi: 10.1016/j.nicl.2017.10.028

Tsubaki, A., Morishita, S., Tokunaga, Y., Sato, D., Tamaki, H., Yamazaki, Y., et al. (2018). Changes in Cerebral Oxyhaemoglobin Levels During and After a Single 20-Minute Bout of Moderate-Intensity Cycling. Oxygen Transport to Tissue XL. Berlin: Springer, 127-131. doi: 10.1007/978-3-319-91287-5_20

Uhlhaas, P. J., and Singer, W. (2006). Neural synchrony in brain disorders: relevance for cognitive dysfunctions and pathophysiology. Neuron 52, 155-168. doi: 10.1016/j.neuron.2006.09.020

Vasques, P. E., Moraes, H., Silveira, H., Deslandes, A. C., and Laks, J. (2011). Acute exercise improves cognition in the depressed elderly: the effect of dual-tasks. Clinics 66, 1553-1557. doi: 10.1590/S1807-59322011000900008

Vaynman, S., Ying, Z., and Gomez-Pinilla, F. (2004). Hippocampal BDNF mediates the efficacy of exercise on synaptic plasticity and cognition. Eur. J. Neurosci. 20, 2580-2590. doi: 10.1111/j.1460-9568.2004.03720.x

Venckunas, T., Snieckus, A., Trinkunas, E., Baranauskiene, N., Solianik, R., Juodsnukis, A., et al. (2016). Interval running training improves cognitive flexibility and aerobic power of young healthy adults. J. Strength Condition. Res. 30, 2114-2121. doi: 10.1519/JSC.0000000000001322
Wang, C. C., Alderman, B., Wu, C. H., Chi, L., Chen, S. R., Chu, I. H., et al. (2019). Effects of acute aerobic and resistance exercise on cognitive function and salivary cortisol responses. J. Sport Exerc. Psychol. 41, 73-81. doi: 10.1123/ jsep.2018-0244

Wilke, J., Giesche, F., Klier, K., Vogt, L., Herrmann, E., and Banzer, W. (2019). Acute effects of resistance exercise on cognitive function in healthy adults: a systematic review with multilevel meta-analysis. Sports Med. 49, 905-916. doi: 10.1007/s40279-019-01085-x

Wrann, C. D., White, J. P., Salogiannnis, J., Laznik-Bogoslavski, D., Wu, J., Ma, D., et al. (2013). Exercise induces hippocampal BDNF through a PGC-1 $\alpha /$ FNDC5 pathway. Cell Metab. 18, 649-659. doi: 10.1016/j.cmet.2013.09.008

Yanagisawa, H., Dan, I., Tsuzuki, D., Kato, M., Okamoto, M., Kyutoku, Y., et al. (2010). Acute moderate exercise elicits increased dorsolateral prefrontal activation and improves cognitive performance with Stroop test. Neuroimage 50, 1702-1710. doi: 10.1016/j.neuroimage.2009.12.023

Yang, J., Ruchti, E., Petit, J.-M., Jourdain, P., Grenningloh, G., Allaman, I., et al. (2014). Lactate promotes plasticity gene expression by potentiating NMDA signaling in neurons. Proc. Natl. Acad. Sci. U.S.A. 111, 12228-12233. doi: 10. 1073/pnas.1322912111

Young, J., Angevaren, M., Rusted, J., and Tabet, N. (2015). Aerobic exercise to improve cognitive function in older people without known cognitive impairment. Cochrane Datab. Syst. Rev. 4:CD005381. doi: 10.1002/14651858. CD005381.pub4

Yuen, E. Y., Liu, W., Karatsoreos, I. N., Feng, J., McEwen, B. S., and Yan, Z. (2009). Acute stress enhances glutamatergic transmission in prefrontal cortex and facilitates working memory. Proc. Natl. Acad. Sci. U.S.A. 106, 14075-14079. doi: 10.1073/pnas.0906791106

Zheng, G., Xia, R., Zhou, W., Tao, J., and Chen, L. (2016). Aerobic exercise ameliorates cognitive function in older adults with mild cognitive impairment: a systematic review and meta-analysis of randomised controlled trials. Br. J. Sports Med. 50, 1443-1450. doi: 10.1136/bjsports-2015095699

Zhu, Y., Wu, H., Qi, M., Wang, S., Zhang, Q., Zhou, L., et al. (2018). Effects of a specially designed aerobic dance routine on mild cognitive impairment. Clin. Interv. Aging. 13:1691. doi: 10.2147/CIA.S163067

Zschucke, E., Gaudlitz, K., and Ströhle, A. (2013). Exercise and physical activity in mental disorders: clinical and experimental evidence. J. Prevent. Med. Public Health 46(Suppl. 1), S12-21. doi: 10.3961/jpmph.2013.46. S.S12

Conflict of Interest: The authors declare that the research was conducted in the absence of any commercial or financial relationships that could be construed as a potential conflict of interest.

Copyright (C) 2019 Moriarty, Mermier, Kravitz, Gibson, Beltz and Zuhl. This is an open-access article distributed under the terms of the Creative Commons Attribution License (CC BY). The use, distribution or reproduction in other forums is permitted, provided the original author(s) and the copyright owner(s) are credited and that the original publication in this journal is cited, in accordance with accepted academic practice. No use, distribution or reproduction is permitted which does not comply with these terms. 